NIST Special Publication 1134

\title{
Extended Feature Set Profile Specification
}

Will Chapman

Austin Hicklin

George Kiebuzinski

Peter Komarinski

John Mayer-Splain

Melissa Taylor

Rachel Wallner

http://dx.doi.org/10.6028/NIST.SP.1134

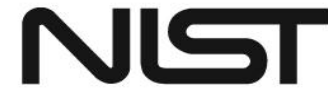

National Institute of Standards and Technology

U.S. Department of Commerce 
NIST Special Publication 1134

\title{
Extended Feature Set Profile Specification
}

\author{
Melissa Taylor \\ Law Enforcement Standards Office \\ Office of Special Programs
}

Will Chapman

Austin Hicklin

George Kiebuzinski

John Mayer-Splain

Rachel Wallner

Noblis

Falls Church, VA

Peter Komarinski

Komarinski \& Associates, LLC

Rotterdam, $N Y$

http://dx.doi.org/10.6028/NIST.SP.1134

January 2013

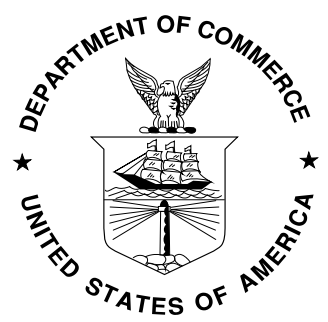

U.S. Department of Commerce Rebecca Blank, Acting Secretary

National Institute of Standards and Technology Patrick D. Gallagher, Under Secretary of Commerce for Standards and Technology and Director 
Certain commercial entities, equipment, or materials may be identified in this document in order to describe an experimental procedure or concept adequately. Such identification is not intended to imply recommendation or endorsement by the National Institute of Standards and Technology, nor is it intended to imply that the entities, materials, or equipment are necessarily the best available for the purpose.

National Institute of Standards and Technology Special Publication 1134 Natl. Inst. Stand. Technol. Spec. Publ. 1134, 11 pages (January 2013) http://dx.doi.org/10.6028/NIST.SP.1134

CODEN: NSPUE2 


\title{
Extended Feature Set Profile Specification
}

January 2013

\begin{abstract}
This specification defines Extended Feature Set (EFS) Profiles-sets of features to be used in latent friction ridge (fingerprint, palmprint, or plantar) searches of automated friction ridge identification systems (AFIS). The EFS Profiles are designed to be interoperable among AFIS systems from different vendors and to enable crossjurisdictional searches that would not otherwise be practical. Multiple EFS Profiles are defined to allow for tradeoffs between examiner time and search accuracy. EFS Profiles also may be used to document latent friction ridge features, such as for potential use in legal proceedings or exchange between latent examiners, and need not be for AFIS matching purposes.

This is a supporting document of the American National Standard for Information Systems: Data Format for the Interchange of Fingerprint, Facial \& Other Biometric Information, American National Standards Institute/National Institute of Standards and Technology, Information Technology Laboratory (ANSI/NIST-ITL) 1-2011. The values defined here are used in field 9.303. Each EFS Profile in this specification defines a subset of the EFS features defined in Type-9 friction ridge feature records.

EFS Profiles are designed so that they may be incorporated by reference into ANSI/NIST-ITL-based application profiles, such as the Latent Interoperability Transmission Specification. For a description of implementation domains and application profiles, see Section 6 of ANSI/NIST-ITL (2011). This decoupling of feature sets from transactions enables different transactions (or transactions from different organizations) to share a common feature set, aiding in interoperability.
\end{abstract}




\section{Contents}

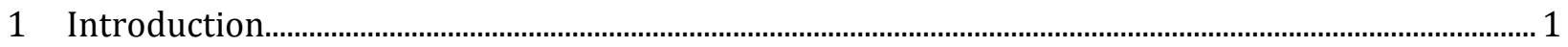

$1.1 \quad$ Purpose

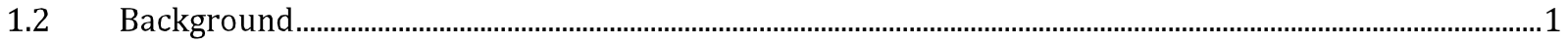

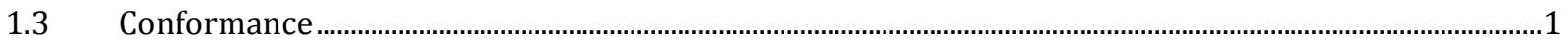

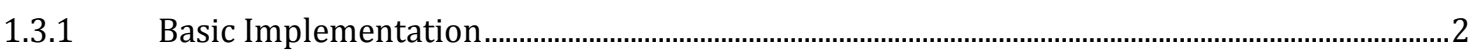

1.3.2 Optional Implementation........................................................................................................2

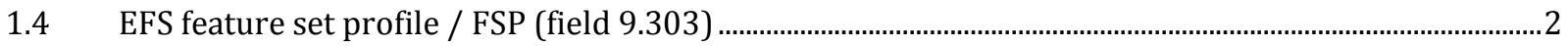

1.5 Correspondence with implementation domains and application profiles ...................................................

1.5.1 Correspondence with the FBI Criminal Justice Information Service Electronic Biometric Transmission Specification ..............................................................................................................

1.5.2 Correspondence with other implementation domains and application profiles.....................

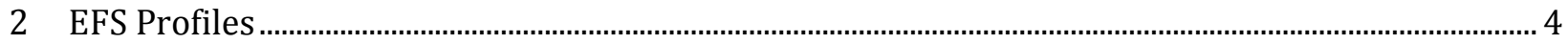

2.1 EFS Profiles for fundamental latent AFIS transactions ......................................................................................... 4

2.1.1 EFS Profile 0: Image-only Profile .................................................................................................

2.1.2 EFS Profile 1: Minimal Markup Profile .............................................................................................

2.1.3 EFS Profile 2: Quick Minutiae Search Profile $\quad$................................................................................

2.1.4 EFS Profile 3: Detailed Markup Profile _.............................................................................................

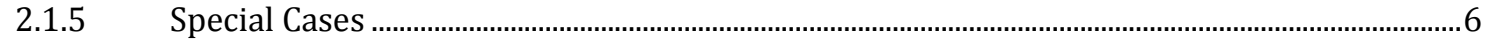

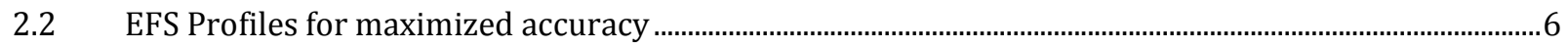

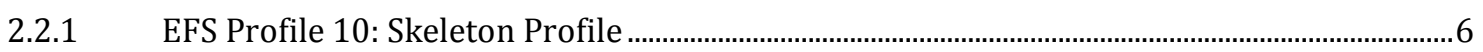

2.2.2 EFS Profile 11: Minutiae Ridge Count Profile........................................................................................

$2.3 \quad$ Special-purpose and legacy profiles ........................................................................................................

2.3.1 EFS Profile 20: Legacy IAFIS Latent Feature Search Profile ........................................................

2.3.2 EFS Profile 21: Quick Minutiae Search Profile Without Image ........................................................

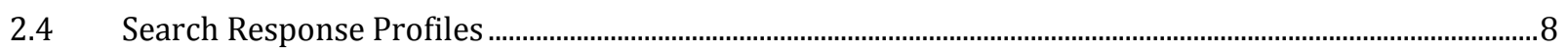

2.4.1 EFS Profile 30: Search Response Profile With All, and Corresponding, Minutiae ...................8

2.4.2 EFS Profile 31: Search Response Profile Without Corresponding Minutiae ..............................

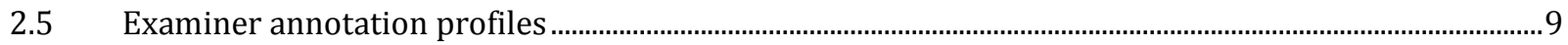

2.5.1 EFS Profile 90: Full Annotation Profile ……………................................................................................

3 Image/region of interest size requirements.............................................................................................. 10 


\section{Introduction}

\subsection{Purpose}

The purpose of Extended Feature Set (EFS) Profiles is to define sets of features to be used in latent friction ridge (fingerprint, palmprint, or plantar ${ }^{1}$ ) searches of automated friction ridge identification systems (AFIS). The EFS Profiles are designed to be interoperable among AFIS systems from different vendors and to enable crossjurisdictional searches that would not otherwise be practical. EFS Profiles also may be used simply to document latent friction ridge features, such as for potential use in legal proceedings or exchange between latent examiners, and need not be for AFIS matching purposes.

While the EFS Profile Specification provides details about which friction ridge features to mark for a given EFS Profile and when to mark them, the Markup Instructions for Extended Friction Ridge Features ${ }^{2}$ provides greater specificity. The Markup Instructions provide instructions specifically for latent print examiners to use in marking extended friction ridge features so that consistency among examiners is maximized.

\subsection{Background}

This is a supporting document of the American National Standard for Information Systems: Data Format for the Interchange of Fingerprint, Facial \& Other Biometric Information, American National Standards Institute/National Institute of Standards and Technology, Information Technology Laboratory 1-2011 (ANSI/NIST-ITL 2011). ${ }^{3}$ This specification defines EFS Profiles for use in field $9.303 .^{4}$

EFS Profiles are defined groups of friction ridge (fingerprint, palmprint, or plantar) features, primarily for use in vendor-neutral latent print searches of AFIS. The friction ridge features used are subsets of the EFS features defined in Type- 9 friction ridge feature records (hereafter referred to as Type-9).

The EFS Profiles defined here are incorporated by reference into the Latent Interoperability Transmission Specification (LITS), ${ }^{5}$ which is an application profile based upon ANSI/NIST-ITL (2011). The EFS Profiles are designed so that they may be incorporated by reference into other application profiles. This decoupling of feature sets from transactions enables different transactions (or transactions from different organizations) to share a common feature set, aiding in interoperability.

In this specification, the term "to mark" refers to the recording of latent friction ridge features (generally by a latent print examiner), and "markup" refers to the features so marked. (ANSI/NIST-ITL (2011) defines the term "mark" as "the point where a needle has pierced the skin, usually associated with drug use," and in its introduction states "The term 'marks' in some nations denotes what is called 'latent prints."')

\subsection{Conformance}

The following terms are used in this specification to indicate mandatory requirements, recommended options, or permissible actions:

- The terms "shall" and "shall not" indicate requirements strictly to be followed to conform to this specification and from which no deviation is permitted.

- The terms "should" and "should not" indicate a recommended or particularly suitable choice if presented with several possibilities, without mentioning or excluding others, or that a certain course of action is preferred but not necessarily required, or that (in the negative form) a certain possibility or course of action is deprecated but not prohibited.

\footnotetext{
${ }^{1}$ Although friction ridge features defined for fingers also may be applicable to toes, they are explicitly excluded from the scope of this document because many of these characteristics, such as pattern classification, are undefined for toes as of this writing.

${ }^{2}$ Markup Instructions for Extended Friction Ridge Features. 2013. http://www.nist.gov/itl/iad/ig/ansi standard.cfm.

${ }^{3}$ ANSI/NIST-ITL. 2011. http://www.nist.gov/itl/iad/ig/ansi standard.cfm.

${ }^{4}$ ANSI/NIST-ITL 8.9.7.4 Field 9.303: EFS feature set profile/FSP.

${ }^{5}$ LITS. 2013. http://www.nist.gov/itl/iad/ig/ansi_standard.cfm.
} 
- The terms "may" and "need not" indicate a course of action permissible within the limits of this specification.

To claim conformance to this specification, implementations shall meet image size requirements as defined in Section 3-Image size requirements of this specification.

ANSI/NIST-ITL Annex $\mathrm{F}^{6}$ is normative for conformance to the standard, as are the instructions for each field defined in the standard for EFS (fields 9.300 through 9.399). For conformance to LITS, the Markup Instructions are normative. Conformance to this specification falls into the following classes:

- Basic Implementation

- Optional Implementation

\subsubsection{Basic Implementation}

An ANSI/NIST-ITL transaction conforms to this specification if it satisfies the requirements for either EFS Profile 0 : Image-only Profile or EFS Profile 2: Quick Minutiae Search.

A system or software (such as AFIS or latent print workstation software) conforms to this specification if it produces ${ }^{7}$ and/or consumes ${ }^{8}$ ANSI/NIST-ITL transactions that satisfy the requirements for both EFS Profile 0 : Image-only Profile and EFS Profile 2: Quick Minutiae Search.

\subsubsection{Optional Implementation}

An ANSI/NIST-ITL transaction conforms to this specification if it satisfies all the Basic Implementation requirements, as well as the requirements for any EFS Profile other than EFS Profile 0: Image-only Profile or EFS Profile 2: Quick Minutiae Search.

A system or software (such as AFIS or latent print workstation software) conforms to this specification if it satisfies all the Basic Implementation requirements, as well as if it either produces ${ }^{7}$ or consumes ${ }^{8}$ ANSI/NIST-ITL transactions that conform to this specification.

\subsection{EFS feature set profile/FSP (field 9.303)}

ANSI/NIST-ITL field 9.303: EFS feature set profile/FSP is defined in this way:

This optional numeric field is used to indicate an EFS Profile, which defines the specific set of EFS fields incorporated in a specific ANSI/NIST-ITL transaction. Profiles can be incorporated by reference into the definition of transactions: this decoupling of feature sets from transactions enables different transactions to share a common feature set, aiding in interoperability. If a given ANSI/NIST-ITL transaction is conformant with two or more profiles, the code for each profile is entered in a separate subfield. The valid values for this field are available in the EFS Profile Specification, which can be downloaded from http://www.nist.gov/it//iad/ig/ansi standard.cfm.

Table 1 defines the valid codes for use in field 9.303.

Table 1: Extended Feature Set Profile Codes

\begin{tabular}{|c|l|c|}
\hline Extended Feature Set Profile Code & \multicolumn{1}{|c|}{ Name } & Image required \\
\hline $0^{9}$ & Image-only Profile & $\mathrm{x}$ \\
\hline 1 & Minimal Markup Profile & $\mathrm{x}$ \\
\hline 2 & Quick Minutiae Search Profile & $\mathrm{x}$ \\
\hline 3 & Detailed Markup Profile & $\mathrm{x}$ \\
\hline $4-9$ & Reserved for future use & \\
\hline
\end{tabular}

${ }^{6}$ EFS Detailed Instructions.

${ }^{7}$ Produces $=$ creates, generates, or exports.

${ }^{8}$ Consumes $=$ parses, ingests, or imports.

${ }^{9}$ Note the code for EFS Profile 0: Image-only Profile is never entered in field 9.303 because no Type- 9 record shall be included in the transaction. 


\begin{tabular}{|c|l|c|}
\hline Extended Feature Set Profile Code & \multicolumn{1}{|c|}{ Name } & Image required \\
\hline 10 & Skeleton Profile & \\
\hline 11 & Minutiae Ridge Count Profile & \\
\hline $12-19$ & Reserved for future use & \\
\hline 20 & Legacy IAFIS* Latent Feature Search Profile & \\
\hline 21 & Quick Minutiae Search Profile without Image & \\
\hline $22-29$ & Reserved for future use & \\
\hline 30 & Search Response Profile with All, and Corresponding, Minutiae & \\
\hline 31 & Search Response Profile without Corresponding Minutiae & \\
\hline $32-89$ & Reserved for future use & \\
\hline 90 & Full Annotation Profile & \\
\hline $91-99$ & Reserved for future use & \\
\hline$*$ Integrated Automated Fingerprint Identification System \\
\hline
\end{tabular}

\subsection{Correspondence with implementation domains and application profiles}

\subsubsection{Correspondence with the FBI Criminal Justice Information Service Electronic Biometric Transmission Specification}

The Federal Bureau of Investigation (FBI) Criminal Justice Information Services (CJIS) Electronic Biometric Transmission Specification (EBTS) ${ }^{10}$ corresponds directly to these EFS profiles:

- Latent Friction Ridge Image Search (LFIS): EFS Profile 0

- Latent Friction Ridge Feature Search (LFFS): EFS Profile 1 (if minutiae are not included)

EFS Profile 2 (if minutiae are included)

EFS Profiles $2+10$ (with skeleton)

EFS Profile 21 (if image is not included)

- Search Results-Latent (SRL):

EFS Profile 30

- Unsolved Biometric Match (ULM): $\quad$ EFS Profile 31

\subsubsection{Correspondence with other implementation domains and application profiles}

A transaction may conform to an implementation domain or application profile other than FBI CJIS EBTS, such as the $L I T S{ }^{10}{ }^{10}$ The organization specifying the implementation domain or application profile should define the EFS Profiles described in this specification that are valid for its purpose(s).

\footnotetext{
${ }^{10} \mathrm{FBI}$ CJIS EBTS. 2011. https://www.fbibiospecs.org/docs/EBTS v9 3 Final\%2012 $07 \quad 11$ clean.pdf.
} 


\section{EFS Profiles}

Each EFS Profile in this specification defines a subset of the EFS features defined in the Type-9 record (fields 9.300 through 9.399). ${ }^{11}$ Two EFS Profiles are designed to be common to, and available across, all interoperable systems: EFS Profile 0: Image-only Profile and EFS Profile 2: Quick Minutiae Search Profile. Additional optional EFS Profiles allow for tradeoffs between examiner markup time and search accuracy, a valuable consideration for systems that can make effective use of the additional features.

Each feature type is accompanied with a summary of when it is appropriate for an image to be marked, both to limit examiner markup and to make explicit for the recipient of a transaction what the absence of a field means.

When the friction ridge image associated with a given Type- 9 record is included in a transaction, it shall be

- $\quad$ an instance of a Type-13, Type-14, Type-15, or Type-19 record

- linked to a Type-9 record by setting the Information Designation Character (IDC) ${ }^{12}$ in field 9.002 and field $x .002$ to the same value (where $x=13,14,15$, or 19)

EFS Profile numbers not specifically listed here are reserved for future use.

\subsection{EFS Profiles for fundamental latent AFIS transactions}

The rationale for these EFS Profiles is to provide four levels of incremental tradeoffs between examiner time and matcher accuracy, based on common use of the features among AFIS vendors:

1. EFS Profile 0: Image-only Profile requires no examiner markup.

2. EFS Profile 1: Minimal Markup Profile requires trivial examiner markup to improve the accuracy of imageonly matching.

3. EFS Profile 2: Quick Minutiae Search Profile includes the friction ridge features common to, and available across, the various AFIS vendors and is compatible with the FBI CJIS EBTS, Department of Defense (DOD) EBTS, ${ }^{13}$ Prüm Convention, ${ }^{14}$ and INTERPOL INT-I ${ }^{15}$ requirements.

4. EFS Profile 3: Detailed Markup Profile includes features that can be used to increase search accuracy in systems that implement this optional profile. Skeletons and ridge counts, which require substantial examiner time, are addressed in the optional EFS Profiles (Section 0).

Each of the EFS Profiles 0 to 3 requires the image to be included in the transaction.

\subsubsection{EFS Profile 0: Image-only Profile}

The image has no obscuring background or multiple impressions and is upright ${ }^{16}$ (visually appears to be within approximately $\pm 15^{\circ}$ of upright).

No Type- 9 record shall be included in the transaction associated with the friction ridge image in the Type-x record (where $x=13,14,15$, or 19). The code for EFS Profile 0: Image-only Profile is never entered in field 9.303 because there is no corresponding Type-9 record.

\subsubsection{EFS Profile 1: Minimal Markup Profile}

The image is accompanied by a Type-9 record with the same IDC value, which shall include the following fields, contingent on the "When to mark" restrictions listed in table 2.

\footnotetext{
${ }^{11}$ Transactions may contain Type- 9 fields outside of the range of EFS features.

${ }^{12}$ For more information concerning IDCs, see Section 7.3.1 of ANSI/NIST-ITL. 2011.

${ }^{13}$ DOD EBTS. 2011. http://www.biometrics.dod.mil/files/documents/standards/dod ebts v3 0.pdf.

${ }^{14}$ Draft Council Decision on the Implementation of Decision 2008/.../JHA on the stepping up of cross-border cooperation, particularly in combating terrorism and cross-border crime. 2008. http://register.consilium.europa.eu/pdf/en/08/st09/st09152-ad01.en08.pdf

${ }^{15}$ Data Format for the Interchange of Fingerprint, Facial \& SMT Information: INTERPOL Implementation. 2011. https://www.interpol.int/Public/Forensic/fingerprints/RefDoc/ImplementationV5.pdf.

${ }^{16}$ This requirement stems from EFS Profile 0: Image-only Profile having no Region of interest/ROI (field 9.300), which accommodates obscuring background or multiple impressions, nor Orientation/ORT (field 9.301), which accommodates non-upright or unknown orientation.
} 
Table 2: EFS Profile 1: Minimal Markup Profile Definition

\begin{tabular}{|c|c|c|c|}
\hline Feature & Field & Mnemonic & When to mark \\
\hline region of interest & 9.300 & ROI & $\begin{array}{l}\text { always (may be identical to the image dimensions); } \\
\text { the ROI polygon/ROP is the fifth information item in field 9.300; it shall be } \\
\text { present in the field if multiple impressions, complex background, or only a } \\
\text { portion of the print is to be searched }\end{array}$ \\
\hline orientation & 9.301 & ORT & $\begin{array}{l}\text { if the impression is upright (visually appears to be within approximately } \\
\pm 15^{\circ} \text { of upright), orientation need not be indicated; if the orientation } \\
\text { cannot be determined, the uncertainty value shall be set to } 180 \text {; otherwise, } \\
\text { the orientation shall be indicated } \\
\text { uncertainty/EUC optional unless set to } 180\end{array}$ \\
\hline $\begin{array}{l}\text { finger, palm, plantar } \\
\text { position }\end{array}$ & 9.302 & FPP & $\begin{array}{l}\text { friction ridge generalized position/FGP-required (unknown finger, palm, or } \\
\text { plantar position may be indicated) } \\
\text { finger segment/FSM-if all or part of the medial or proximal finger } \\
\text { segments are present (fingerprint only) } \\
\text { off-center fingerprint/OCF-if an extreme tip or extreme side (fingerprint } \\
\text { only) }\end{array}$ \\
\hline pattern classification & 9.307 & PAT & if known (does not apply to palms, tips or lower joints or to plantars) \\
\hline cores & 9.320 & COR & if present \\
\hline deltas & 9.321 & DEL & if present \\
\hline no cores present & 9.325 & NCOR & $\begin{array}{l}\text { if no cores are present in the image, set to } Y \text {; otherwise, field shall not be } \\
\text { used }\end{array}$ \\
\hline no deltas present & 9.326 & NDEL & $\begin{array}{l}\text { if no deltas are present in the image, set to } Y \text {; otherwise, field shall not be } \\
\text { used }\end{array}$ \\
\hline
\end{tabular}

\subsubsection{EFS Profile 2: Quick Minutiae Search Profile}

The image is accompanied by a Type-9 record with the same IDC value, which shall include the following fields (in addition to the features specified in EFS Profile 1: Minimal Markup Profile), contingent on the "When to mark" restrictions listed in table 3.

Table 3: EFS Profile 2: Quick Minutiae Search Profile Definition

\begin{tabular}{|c|c|c|c|}
\hline Feature & Field & Mnemonic & When to mark \\
\hline \multicolumn{4}{|c|}{ (all features from Minimal Markup Profile) } \\
\hline minutiae & 9.331 & MIN & $\begin{array}{l}\text { if present all minutiae shall be marked (inclusive of short ridges, short } \\
\text { enclosures, and areas of high curvature) }\end{array}$ \\
\hline no minutiae present & 9.334 & NMIN & if not present (an unusual case), set to $Y$; otherwise, field shall not be used \\
\hline evidence of fraud & 9.354 & EOF & if present \\
\hline tonal reversal & 9.314 & TRV & if entire impression is inverted black for white \\
\hline
\end{tabular}

Where there is evidence of fraud $^{17}$ (evasion, spoofing, forged evidence, and fabricated evidence), it shall be indicated. This information may be used in the administration of an AFIS database and may or may not be not be used by the AFIS matcher itself.

If the entire impression is inverted black for white so that ridges are shown in white and valleys are shown in black, that should be indicated in the Tonal reversal/TRV (field 9.314); this field is not used if only a portion of the impression is tonally inverted. ${ }^{18}$

\subsubsection{EFS Profile 3: Detailed Markup Profile}

The image is accompanied by a Type-9 record with the same IDC value, which shall include the following fields (in addition to the features specified in EFS Profile 2: Quick Minutiae Search Profile), contingent on the "When to mark" restrictions listed in table 4.

Table 4: EFS Profile 3: Detailed Markup Profile Definition

\begin{tabular}{|c|c|c|c|}
\hline Feature & Field & Mnemonic & When to mark \\
\hline \multicolumn{4}{|c|}{ (all features from Minimal Markup Profile and Quick Minutiae Search Profile) } \\
\hline ridge quality/confidence map & 9.308 & RQM & always \\
\hline ridge flow map & 9.310 & RFM & always \\
\hline
\end{tabular}

\footnotetext{
${ }^{17}$ For more information concerning EFS code for fraud type assessment, see ANSI/NIST-ITL Table 50 EFS codes for fraud type assessments.

${ }^{18}$ ANSI/NIST-ITL 8.9.7.12 Field 9.314: EFS tonal reversal/TRV.
} 


\begin{tabular}{|c|c|c|c|}
\hline Feature & ield & Mnemonic & When to mark \\
\hline center point of reference & 9.323 & CPR & if known (does not apply to palms or plantars) \\
\hline distinctive features & 9.324 & DIS & $\begin{array}{l}\text { if scars or other physical abnormalities (e.g., warts or } \\
\text { dysplasia) are present; other distinctive features optional }\end{array}$ \\
\hline dots & 9.340 & DOT & if present \\
\hline incipient ridges & 9.341 & INR & if present \\
\hline no dots present & 9.346 & NDOT & if not present, set to $Y$; otherwise, field shall not be used \\
\hline no incipient ridges present & 9.347 & NINR & if not present, set to $Y$; otherwise, field shall not be used \\
\hline core-delta ridge counts & 9.322 & CDR & $\begin{array}{l}\text { if both core(s) and delta(s) are present, may be marked } \\
\text { optionally if either a core or a delta is present, with a } \\
\text { minimum ridge count }\end{array}$ \\
\hline
\end{tabular}

\subsubsection{Special Cases}

In the unusual circumstance that the friction ridge impression is believed to have changed size or scale from potential comparisons, such as for deceased subjects with swollen or desiccated skin, or when comparing adult and juvenile prints, that should be indicated in the Possible growth or shrinkage/PGS (field 9.317). ${ }^{19}$ This field may be used optionally with EFS Profiles 0-3.

Possible lateral reversal/PLR (field 9.315$)^{20}$ is used to indicate that it is unclear if the image may be flipped left for right, such as in some prints on transparent tape. The purpose of the field is to indicate that the recipient of the transaction should compare (or match) the image both forward and reversed left for right. This field may optionally be used with EFS Profile 90: Full Annotation Profile when annotating casework. The PLR field is not used for AFIS searches. When an impression that may be a possible lateral reversal is used in EFS Profiles 0-3, then two separate and distinct search transactions shall be created, one forward and one flipped, to avoid the complexity/potential confusion of commingling candidate matches for normal and flipped images in a single response; the Possible lateral reversal/PLR (field 9.315) shall not be used when EFS Profiles 0-3 are used.

\subsection{EFS Profiles for maximized accuracy}

These EFS Profiles include features that require substantial examiner time but may optionally be used to increase search accuracy in systems that implement this optional profile (such as for critical cases or poor-quality latents).

These EFS Profiles are to be combined ${ }^{21}$ with the EFS Profiles for fundamental latent AFIS transactions (EFS Profiles 1-3); where these EFS Profiles are used, field 9.303 shall contain both the EFS Profiles for fundamental latent AFIS transactions code (EFS Profiles 1-3) and the code for the optional EFS Profile. For example, a skeleton in combination with the EFS Profile 1: Minimal Markup Profile would include the codes 1 and 10 as separate subfields (occurrences) within field 9.303; a skeleton in combination with the EFS Profile 3: Detailed Markup Profile would include the codes 3 and 10 as separate subfields (occurrences) within field 9.303.

\subsubsection{EFS Profile 10: Skeleton Profile}

The Skeletonized image/SIM (field 9.372), listed in table 5, shall be included in addition to the fields in the specified EFS Profiles 1-3.

Table 5: EFS Profile 10: Skeleton Profile Definition

\begin{tabular}{|l|l|l|l}
\multicolumn{1}{c}{ Feature } & Field & Mnemonic & When to mark \\
\begin{tabular}{|l|l|l|l} 
(all features from the specified profile 1 to 3$)$ & & \\
skeletonized image & 9.372 & SIM & always
\end{tabular}
\end{tabular}

When EFS Profile 10: Skeleton Profile is used in combination with EFS Profile 3: Detailed Markup Profile, the skeleton is marked as follows with respect to the ridge quality map/RQM (field 9.308):

- In all areas marked as "definitive minutiae" (Green) or better, the skeleton must be complete and unbroken, and the skeleton's ridges stop only at ridge endings. Any areas of the print that cannot be represented in the skeleton shall be marked as "debatable minutiae" (Yellow) or worse.

\footnotetext{
${ }^{19}$ ANSI/NIST-ITL 8.9.7.15 Field 9.317: EFS possible growth or shrinkage/PGS.

${ }^{20}$ ANSI/NIST-ITL 8.9.7.13 Field 9.315: EFS possible lateral reversal/PLR.

${ }^{21}$ The maximum number of EFS Profiles that may be contained in a single transaction is nine.
} 
- In areas marked as "debatable minutiae" (Yellow), the skeleton may be incomplete, and the skeleton's ridges may stop at locations that do not correspond to ridge endings.

When EFS Profile 10: Skeleton Profile is used in combination with EFS Profile 1: Minimal Markup Profile or EFS Profile 2: Quick Minutiae Search Profile, treatment of the skeleton is as if the entire ROI had been marked using the ridge quality map value of "debatable minutiae" (Yellow): the skeleton may be incomplete, and the skeleton's ridges may stop at locations that do not correspond to ridge endings.

\subsubsection{EFS Profile 11: Minutiae Ridge Count Profile}

Except in the case when no minutiae present/NMIN (field 9.334), minutiae ridge counts/MRC (field 9.333), listed in table 6, shall be included in addition to the fields in the specified EFS Profile 2: Quick Minutiae Search Profile or EFS Profile 3: Detailed Markup Profile-since ridge counts are dependent on minutiae, they would not apply to EFS Profile 0: Image-only Profile or EFS Profile 1: Minimal Markup Profile. The minutiae ridge count algorithm shall be indicated in field 9.332 .

Table 6: EFS Profile 11: Minutiae Ridge Count Profile Definition

\begin{tabular}{|c|c|c|c|}
\hline Feature & Field & Mnemonic & When to mark \\
\hline \multicolumn{4}{|c|}{ (all features from the specified profile 1 to 3 ) } \\
\hline minutiae ridge count algorithm & 9.332 & MRA & always \\
\hline minutiae ridge counts & 9.333 & $\mathrm{MRC}$ & always \\
\hline
\end{tabular}

\subsection{Special-purpose and legacy profiles}

These profiles are specified for certain special cases as described below.

\subsubsection{EFS Profile 20: Legacy IAFIS Latent Feature Search Profile}

This EFS Profile accommodates the fields used in the FBI Electronic Fingerprint Transmission Specification (EFTS) 7.1 LFFS transaction definition, as used by the IAFIS and implemented as an interface for some other systems. This EFS Profile does not require the image to be present. (See table 1 for which EFS Profiles require an image.) This EFS Profile does not apply to palms, tips, lower joints, or plantars. The Type-9 record shall include the following fields, contingent on the "When to mark" restrictions listed in table 7.

Table 7: EFS Profile 20: Legacy IAFIS Latent Feature Search Profile Definition

\begin{tabular}{|c|c|c|c|}
\hline Feature & Field & Mnemonic & When to mark \\
\hline region of interest & 9.300 & $\mathrm{ROI}$ & $\begin{array}{l}\text { if multiple impressions, complex background, or only a portion } \\
\text { of the print is to be searched }\end{array}$ \\
\hline orientation & 9.301 & ORT & if known \\
\hline finger, palm, plantar position & 9.302 & FPP & $\begin{array}{l}\text { friction ridge generalized position/FGP-required, unknown } \\
\text { finger, palm, plantar position may be indicated } \\
\text { finger segment/FSM-not used } \\
\text { off-center fingerprint/OCF-not used }\end{array}$ \\
\hline pattern classification & 9.307 & PAT & if known \\
\hline cores & 9.320 & COR & if present \\
\hline deltas & 9.321 & DEL & if present \\
\hline core-delta ridge counts & 9.322 & CDR & if one or more cores and one or more deltas are present \\
\hline minutiae & 9.331 & MIN & if present \\
\hline minutiae ridge count algorithm & 9.332 & MRA & if minutiae are present (set to "EFTS7") \\
\hline minutiae ridge counts & 9.333 & $\mathrm{MRC}$ & if minutiae are present \\
\hline
\end{tabular}

\subsubsection{EFS Profile 21: Quick Minutiae Search Profile Without Image}

There are occasionally situations in which it is not possible to include the latent image in a search due to bandwidth restrictions (possibly in emergency situations) or sensitivity of images. Use of this EFS Profile is not considered best practice with respect to accuracy and shall be used only if specifically called for. This EFS Profile is identical to the EFS Profile 2: Quick Minutiae Search Profile with the exception that EFS Profile 21: Quick Minutiae Search Profile Without Image shall not include an image in the transaction. (See table 1 for which EFS Profiles require an image.) 


\subsection{Search Response Profiles}

These EFS profiles define the use of fields in the range 9.300 through 9.399 in a Type-9 record if features are returned in association with candidate lists, such as in a search results transaction in response to a latent AFIS search. Most frequently the candidates are exemplars (rolled or plain impressions) but may be latents from a search of unsolved latent databases.

Note that this does not mandate that features necessarily be returned for search results transactions; it defines the contents when search results transactions do include such features.

\subsubsection{EFS Profile 30: Search Response Profile With All, and Corresponding, Minutiae}

The search response returns the pattern classification, friction ridge position, and all minutiae for the candidate; in addition, the specific minutiae that correspond between the search print and the candidate are indicated in the corresponding points or features field, if present. The Type- 9 record shall include the following fields, contingent on the "When to mark" restrictions listed in table 8.

Table 8: EFS Profile 30: Search Response Profile With All, and Corresponding, Minutiae Definition

\begin{tabular}{|c|c|c|c|}
\hline Feature & Field & Mnemonic & When to mark \\
\hline region of interest & 9.300 & ROI & always \\
\hline orientation & 9.301 & ORT & if known \\
\hline finger, palm, plantar position & 9.302 & FPP & $\begin{array}{l}\text { friction ridge generalized position/FGP-required, unknown } \\
\text { finger, palm, plantar position may be indicated } \\
\text { finger segment/FSM-if all or part of the medial or proximal } \\
\text { finger segments are present (fingerprint only) } \\
\text { off-center fingerprint/OCF-if an extreme tip or extreme side } \\
\text { (fingerprint only) }\end{array}$ \\
\hline pattern classification & 9.307 & PAT & $\begin{array}{l}\text { if known (does not apply to palms, tips, or lower joints or to } \\
\text { plantars) }\end{array}$ \\
\hline cores & 9.320 & COR & if present \\
\hline deltas & 9.321 & DEL & if present \\
\hline no cores present & 9.325 & NCOR & if not present, set to $Y$; otherwise, field shall not be used \\
\hline no deltas present & 9.326 & NDEL & if not present, set to $Y$; otherwise, field shall not be used \\
\hline minutiae & 9.331 & $\mathrm{MIN}$ & if present \\
\hline no minutiae present & 9.334 & NMIN & if not present, set to $Y$; otherwise, field shall not be used \\
\hline method of feature detection & 9.350 & MFD & recommended (vendor and version of algorithm) \\
\hline corresponding points or features & 9.361 & $\mathrm{CPF}$ & if present \\
\hline $\begin{array}{l}\text { relative rotation of corresponding } \\
\text { print }\end{array}$ & 9.363 & $\mathrm{RRC}$ & if known \\
\hline
\end{tabular}

\subsubsection{EFS Profile 31: Search Response Profile Without Corresponding Minutiae}

The search response returns the pattern classification, friction ridge position, and all minutiae for the candidate but no corresponding points or features. The Type-9 record shall include the following fields, contingent on the "When to mark" restrictions listed in table 9.

Table 9: EFS Profile 31: Search Response Profile Without Corresponding Minutiae Definition

\begin{tabular}{|c|c|c|c|}
\hline Feature & Field & Mnemonic & When to mark \\
\hline region of interest & 9.300 & $\mathrm{ROI}$ & always \\
\hline orientation & 9.301 & ORT & if known \\
\hline finger, palm, plantar position & 9.302 & FPP & $\begin{array}{l}\text { friction ridge generalized position/FGP-required, unknown } \\
\text { finger, palm, plantar position may be indicated } \\
\text { finger segment/FSM-if all or part of the medial or proximal } \\
\text { finger segments are present (fingerprint only) } \\
\text { off-center fingerprint/OCF-if an extreme tip or extreme side } \\
\text { (fingerprint only) }\end{array}$ \\
\hline pattern classification & 9.307 & PAT & $\begin{array}{l}\text { if known (does not apply to palms, tips, or lower joints or to } \\
\text { plantars) }\end{array}$ \\
\hline cores & 9.320 & COR & if present \\
\hline deltas & 9.321 & DEL & if present \\
\hline minutiae & 9.331 & $\mathrm{MIN}$ & always \\
\hline no cores present & 9.325 & NCOR & if not present, set to $Y$; otherwise, field shall not be used \\
\hline
\end{tabular}




\begin{tabular}{|l|l|l|l|}
\hline \multicolumn{1}{|c|}{ Feature } & Field & Mnemonic & \\
\hline no deltas present & 9.326 & NDEL & if not present, set to Y; otherwise, field shall not be used \\
\hline no minutiae present & 9.334 & NMIN & if not present, set to Y; otherwise, field shall not be used \\
\hline method of feature detection & 9.350 & MFD & recommended (vendor and version of algorithm) \\
\hline
\end{tabular}

\subsection{Examiner annotation profiles}

These EFS Profiles are included solely for examiner annotation of analysis or comparison and shall not be used for AFIS searches.

\subsubsection{EFS Profile 90: Full Annotation Profile}

The image is accompanied by a Type-9 record with the same IDC value, which may optionally include any of the extended feature set fields, such as pores; creases; ridge edge features; latent substrate, matrix, or processing; possible lateral reversal; or analysis assessment (value). This is a superset of EFS Profile 2: Quick Minutiae Search Profile. At a minimum, the features specified in the EFS Profile 2: Quick Minutiae Search Profile shall be marked according to the rules in that profile. 


\section{Image/region of interest size requirements}

This section defines the maximum size of the ROI for EFS Profiles 1-3, and the maximum size of the associated friction ridge image for EFS Profile 0: Image-only Profile. Maximum sizes apply only to fingerprints or extreme tips, ${ }^{22}$ (not to palmprints, lower joints, full finger views, or plantars).

- For EFS Profiles 1, 2, or 3, the maximum width or height of the ROI shall not exceed 1.0" (25.4 mm); there is no maximum image size.

- For EFS Profile 0 (which has no associated ROI), the maximum width or height of the image saved in the associated Type-13 or Type-14 record shall not exceed $1.0^{\prime \prime}(25.4 \mathrm{~mm})$.

For palmprints, lower joints, full finger views, or plantars, there is no maximum image or ROI size beyond the ANSI/NIST-ITL limitations; however, best practices may choose to constrain the image or ROI size.

A system or software (such as AFIS or latent print workstation software) that conforms to this specification and produces ${ }^{23}$ ANSI/NIST-ITL transactions shall not permit larger image or ROI sizes than specified above.

A system or software (such as AFIS or latent print workstation software) that conforms to this specification and consumes ${ }^{24}$ ANSI/NIST-ITL transactions may permit larger image or ROI sizes than specified above.

${ }^{22}$ Applies only to fingerprint record types.

${ }^{23}$ Produces $=$ create, generate, or export.

${ }^{24}$ Consumes $=$ parse, ingest, or import. 